\title{
Das andere Bali II: Urbanisierung auf der Insel der Götter
}

\section{Einleitung: Balibild und Urbanisierung}

Schon zur Kolonialzeit schufen westliche Forscher - darunter auffallend viele Kolonialbeamte - das bis heute bestehende, stark idealisierende Bild einer Insel mit einzigartiger, kosmologisch verankerter Kultur.' Seitdem ist das Image Balis in der Weltöffentlichkeit von exotischen Tempelritualen mit Tänzerinnen und Tänzern vor idyllischer, ländlicher Kulisse geprägt. Weniger exotische Aspekte der balinesischen Gesellschaft wie Urbanisierung (und deshalb auch Stadtforschung) sind demgegenüber sowohl in der Kolonial- als auch Nachkolonialzeit mehr als stiefmütterlich behandelt worden. ${ }^{2}$

Scheint das Defizit an Stadtforschung während der Kolonialzeit beim damaligen geringen Verstädterungsgrad Balis noch einigermaßen begreiflich, kann es heute nicht mehr akzeptiert werden. So wohnten 1930 nur gerade 2,3\%, im Jahre 1989 hingegen schon 19\% der Inselbewohner in Städten. ${ }^{3}$ Auch das "paradiesische» Bali ist somit vom global zu konstatierenden Städtewachstum nicht verschont worden. Sichtbarster Ausdruck davon ist die Provinzhauptstadt Denpasar, die 1989 als vierzehntgrößte Stadt Indonesiens mit knapp 315000 mehr Einwohner als alle anderen Städte auf der Insel insgesamt aufwies. ${ }^{4}$

Im vorliegenden Aufsatz ist deshalb der Blick für einmal nicht auf die in der Baliforschung dominierenden Bereiche der Kultur und Kunst gerichtet. Er will vielmehr mit der Analyse spezifischer Aspekte des Urbanisierungsprozesses in Denpasar einen Beitrag zum Verständnis des "anderen» Bali leisten. ${ }^{5}$ Thematisch stehen Transformationsprozesse des endogenen Sozialsystems im Spannungsfeld von staatlichem Handeln und urbanem Kontext im Mittelpunkt. Damit soll versucht werden, einen ersten Entwurf zur Analyse der urbanen Variante des balinesischen Sozialsystems zu leisten.

Jede Diskussion institutionellen Wandels muß sich - soweit rekonstruierbar - an der Ausgangslage, dem endogenen Sozialsystem, seinen Institutionen und seinem handlungsleitenden Werte- und Normensystem orientieren. Letzteres wird in Bali, wie in ganz Indonesien, meist unter dem Begriff adat subsumiert. ${ }^{6}$ Adat - häufig sehr vereinfachend mit «Handeln gemäß Überlieferung» (customary practice) oder "Gewohnheitsrecht" übersetzt steht ursprünglich für die gemeinsamen sozialen, rechtlichen, moralischen und religiösen Beziehungen, wie sie von den Vorfahren übermittelt worden sind (WARREN 1990: 1/2)
Unter der Vielzahl von adatbestimmten Regeln und Einrichtungen zur Steuerung der balinesischen Gesellschaft, in denen das adat als gelebte Realität bewahrt und weitergegeben werden soll, interessieren hier die zwei wichtigsten Basisinstitutionen: die Organisationsformen der Dorfgemeinschaft (desa) und ihrer "Unterabteilungen", der Nachbarschaftsvereinigungen (banjar).? In ihrer ursprünglichen und heute nur noch idealtypisch gültigen Ausprägung sind desain erster Linie für Beziehungen der Dorfbewohner zur unsichtbaren Welt der Götter und gottgewordenen Vorfahren zuständig und regeln so das spirituelle und materielle Gleichgewicht innerhalb des Dorfterritoriums. In den (kleineren) banjar hingegen ist die gegenseitige Hilfe unter Nachbarn institutionalisiert, womit sie vor allem für die profane Sphäre der Beziehungen der Dorfbewohner untereinander zuständig sind. ${ }^{8}$

Die Forschung zu desa und banjar konzentrierte sich bis vor kurzem vor allem auf ihre ursprünglichen oder «traditionellen" Adatausprägungen. Erst in jüngerer Zeit wird das dynamische Wechselspiel zwischen staatlichem Handeln und lokaler Tradition thematisiert. ${ }^{9}$ Alle bisherigen Studien zum institutionellen Wandel im allgemeinen sowie zu desa und banjar im speziellen sind im ländlichen Raum angesiedelt, während noch keine Untersuchungen für städtische Kontexte vorliegen. Dies ist zum Teil verständlich, sind doch beide Institutionen die konstituierenden Elemente einer ursprünglich ländlichen Gesellschaftsstruktur. Der Begriff kota adat (Adatstadt), wie er für die Gemeinschaft der einheimischen Bewohner der Stadt Denpasar eingeführt wird, ist denn auch eine Neuschöpfung.

Die Zusammensetzung der Stadtbevölkerung von Denpasar hat sich seit der vorkolonialen Zeit massiv verändert: 1986 umfaßte sie lediglich noch 57\% «Einheimische»; die übrigen Bewohner setzten sich aus $23 \%$ zugewanderten Balinesen und 20\% Nichtbalinesen zusammen. ${ }^{10}$ Immerhin $43 \%$ der Stadtbevölkerung stellen also das zentrale "Axiom» des idealtypischen adat und seiner Institutionen - von einheimischen Vorfahren überlieferte Handlungsanleitungen und deshalb an direkte Nach-

Andreas Tarnutzer, dipl. Geograph,

Geograph. Institut der Universität Zürich Irchel, Winterthurerstraße 190, 8057 Zürich 
kommen und Lokalität gebundener Gültigkeitsbereich ganz oder teilweise in Frage und zwingen zu Anpassungen.

Für die Untersuchung eines städtischen Raumes ist deshalb die Analyse der Transformationsprozesse zentral, die sich in den endogenen, ehemals an dörflichen Mustern orientierten Institutionen des balinesischen Sozialsystems abspielen. Im Detail betrifft dies Fragen nach der Aufnahme- und Wandlungsfähigkeit bestehender lokaler desa und banjar sowie nach Ursachen und Bedingungen allenfalls stattfindender Neugründungen. Dies soll Rückschlüsse auf grundlegende Prozesse im Schnittpunkt von endogenem Sozialsystem, Urbanisierung und staatlichem Handeln erlauben. Es kann davon ausgegangen werden, daß der Anteil der städtischen Bevölkerung in Zukunft weiter wachsen wird, weshalb postuliert wird, daß der hier im Zentrum stehenden größten Stadt Denpasar die Funktion eines «Laboratoriums» zukommt, welches den Trend zukünftiger Entwicklungen des balinesischen Sozialsystems aufzeigt.

Im folgenden werden zuerst die wesentlichen Entwicklungsschritte von Denpasar kurz dargestellt. AnschlieBend werden einige Überlegungen zum adat und seinen Institutionen desa und banjar im Spannungsfeld zwischen staatlichem Handeln und städtischem Kontext vorgestellt. Darauf aufbauend werden quantitative und qualitative Veränderungen der desa und banjar innerhalb des heutigen Denpasar diskutiert, die abschließend interpretiert werden.

\section{Entwicklungsstufen der Stadt Denpasar}

Über die vorkoloniale Hauptstadt des südbalinesischen Reiches Badung ist wenig bekannt. Die binodale Siedlung gleichen Namens, die sich um die zwei Hauptpaläste des regierenden Fürstenhauses beidseits des Flusses Badung gruppierte, war das magisch-spirituelle, politische und räumliche Zentrum ihres Stadtstaates (city state ${ }^{\prime \prime}$ ) und unterschied sich anscheinend weder in Größe noch Habitus von Zentren anderer Fürstentümer auf Bali.

Als niederländische Kolonialtruppen 1906 Badung als eines der letzten balinesischen Reiche eroberten, marschierten gegen 7000 in festlichem Ornat gekleidete und mehrheitlich nur mit kris und Lanzen bewaffnete Badunger geradeaus ins Feuer der holländischen Gewehre, die mehr als 1000 Männer, Frauen und Kinder niederstreckten. ${ }^{12}$ Da bereits damals Kriegsberichterstatter und Fotoreporter das Expeditionsheer begleiteten, ist die Geschichte dieses spektakulären Massensuizids (puputan ${ }^{13}$ ) um die Welt gegangen und hat Bali ein erstes Mal ins Bewußtsein einer weiteren Öffentlichkeit gerückt.

Ohne das fremde Element der Kolonisation wäre die nun nach einem der beiden Hauptpaläste des regierenden Fürstenhauses in Denpasar umbenannte Siedlung wohl bis heute nicht mehr als eine balinesische Kleinstadt unter anderen. Seit sie als koloniales Zentrum von Südbali ausgebaut wurde, scheint ihr Weg vorgezeichnet: Verwaltungs-, bald darauf Handels- und wenig später
Touristenzentrum machten bereits die Kolonialstadt zum "Zürich» von Bali, während der alten "Rivalin» Singaraja im Norden der Insel als Sitz der Provinzverwaltung die Funktion eines balinesischen «Bern" verblieb. Weitere Impulse für die Stadtentwicklung brachte die Zeit der japanischen Besetzung und des anschließenden Unabhängigkeitskampfes, in der die Siedlung erstmals zentralörtliche Funktionen für die ganze Insel erfüllte. Ihre Dominanz wurde 1960 mit der Verlegung der Provinzhauptstadt von Singaraja nach Denpasar zusätzlich verstärkt. In dieser Zeit sprengte die Stadt ihre während der ganzen Kolonialzeit nicht wesentlich veränderten Grenzen, und erste suburbane Entwicklungen machten sich in den unmittelbar anschließenden Dörfern bemerkbar. Die Bevölkerung der Kernstadt - die kota der Kolonialzeit - wuchs jedoch noch ein Jahrzehnt lang schneller als die ihres Hinterlandes. Seit der Etablierung der "Neuen Ordnung» (orde baru) haben sich Zentralisierungs- und Vereinheitlichungstendenzen im administrativen Bereich durch Regional- und Dorfadministrationsgesetze weiter verstärkt und hat gleichzeitig der behördliche Druck auf die Adatinstitutionen zugenommen.

Citybildung und parallele Suburbanisierung setzten erst ein, nachdem die orde baru die indonesische Wirtschaft auf Expansionskurs getrimmt hatte und 1969 der internationale Flughafen unweit von Denpasar eröffnet worden war. Nun legten sowohl die Investitionen aus javanischen Großstädten als auch die Touristenzahlen kräftig zu. Dies bedeutete den Anfang der bis heute anhaltenden Karriere des balinesischen Verwaltungs-, Dienstleistungs- und Handelszentrums zu einer der boom towns von Indonesien.

1978 trug auch die Zentralverwaltung dieser Entwicklung Rechnung. Die Eingemeindung von 13 umliegenden Dörfern bewirkte eine beträchtliche Ausweitung des Stadtgebietes, und mit der gleichzeitigen Gewährung des Status einer kota administratif wurde Denpasar erstmals eine - allerdings politisch und finanziell unselbständige - administrative Einheit. Erst 1992 ist es schließlich zur kotamadya geworden und kann sich somit als «Region 2. Ordnung» (daerah tingkat II) in beschränktem Maße selbst verwalten.

\section{Adat und staatliches Handeln}

Sowohl Dorf-(desa-) wie auch Nachbarschafts-(banjar-) Organisationen sind in diesem Jahrhundert zuerst vom Kolonialstaat und später vom Nationalstaat grundlegend umgeformt worden und haben damit einen entscheidenden Bedeutungswandel erlebt. Die Kolonialverwaltung baute ein ihren Bedürfnissen entsprechendes Verwaltungssystem auf, in welchem desa und banjar die beiden untersten Stufen bildeten. Diese modernen Varianten von desa und banjar wurden später als desa dinas und banjar dinas ${ }^{14}$ bezeichnet. Damit hatten die vorkolonialen Basisinstitutionen ihre umfassende Bedeutung für das soziale Leben in der Dorfgemeinschaft verloren, eine erste fundamentale Funktionsveränderung, deren Auswirkungen auch heute noch gerne ignoriert werden. Die 
ursprünglichen desa und banjar existierten zwar weiterhin, waren von nun an allerdings hauptsächlich auf ihre magisch-religiöse Funktion beschränkt und damit aus den wesentlichen politischen Strukturen und Entscheidungsmechanismen ausgeschlossen. Sie waren nun Teil des im ganzen Kolonialreich als Antithese zur Moderne geschaffenen, "zeitlosen" und "traditionellen» adat-Bereichs geworden. Zur Abgrenzung gegenüber ihren administrativen Schwesterinstitutionen wurden deshalb die funktionell "reduzierten" Dorfgemeinschaften jetzt als desa adat und deren Unterabteilungen als banjar adat bezeichnet.

Mit der Beibehaltung des um seine «weltlichen» Funktionen reduzierten adat und seiner Institutionen schuf sich der Kolonialstaat ein Gefäß, welches die Kontinuität des «traditionellen» dörflichen Sozialsystems und damit der indonesischen (hier: balinesischen) Kultur gewährleisten sollte. Der indonesische Nationalstaat hat diese Idee des adat als Bollwerk gegen "moderne» westliche Einflüsse als ideologisches Programm weitergeführt und ausgebaut. Insbesondere nachdem in den 70er Jahren die politisch-administrative Konsolidierung des gesamten Staatsgebietes erreicht worden war, ist der staatliche Einfluß auf das adat immer dominanter geworden. ${ }^{15}$

\section{Adat im städtischen Kontext}

$\mathrm{Zu}$ Unrecht in der balispezifischen Diskussion bis anhin weitgehend vernachlässigt, bieten Urbanisierungsprozesse und städtische Siedlungen eine Reihe interessanter Ansatzpunkte für Untersuchungen institutionellen Wandels. Dies liegt zum einen an der Tatsache, daß die frühen Städte, d.h. die Siedlungen um die Paläste (puri) der wichtigsten Fürsten, nicht nur in ihrem Habitus, sondern auch in den hier interessierenden Elementen ihrer Sozialorganisation grundsätzlich ländlicher und damit dörflicher Natur waren. Alle vorhandenen Indizien deuten darauf hin, daß sich vorkoloniale "städtische" banjaı oder desa in Funktion und Struktur nicht wesentlich von ihren «dörflichen» Pendants unterschieden. Erst mit der Kolonisation kamen «fremde Elemente» zum Tragen, die einen Funktions- und Strukturwandel der wichtigsten Zentren bewirkten, von denen sich einige, insbesondere aber Denpasar und Singaraja, zu eigentlichen Kolonialstädten entwickelten.

Ausgelöst durch das explosionsartige Wachstum der Stadt ab 1970, stellt das Phänomen der Zuwanderung spezielle Herausforderungen für das adat und seine Institutionen. Denn sie stellt einen zentralen Aspekt der Adatinstitutionen in Frage, nämlich ihren ausgeprägt lokalen Charakter, der von ihrer Funktion als Basis aller Beziehungen der gegenwärtigen Generation zu ihren Vorfahren einerseits und für das Zusammenleben der "Einheimischen" andererseits herrührt. Zuwanderer verlassen ja ihr altes Dorfgebiet und damit die Lokalität ihrer eigenen Vorfahren und ziehen in ein Gebiet, das bereits von autochthonen Vorfahren und Gemeinschaftsinstitutionen "besetzt" ist. Was ihre Adatbeziehungen betrifft, sind sie also zugleich Auswanderer und Einwande- rer oder, pointierter formuliert, sowohl «Renegaten» wie Eindringlinge.

\section{Adat, städtischer Kontext und staatliches Handeln}

Während staatliche Strategien zur Vereinheitlichung von desa adat nicht explizit auf den städtischen Kontext ausgerichtet waren - ja die diesbezüglich stattfindenden Veränderungen größtenteils ignorierten ${ }^{16}$ - war dies bei banjar keineswegs der Fall. Als der Stadt Denpasar 1978 der Status einer kota administratif zugesprochen wurde, war anscheinend genügend Handlungsbedarf geschaffen, um sich grundsätzlich den institutionellen Aspekten der Integration neuer Stadtbewohner - sowohl balinesischer als auch der von anderen Inseln stammenden - zu widmen.

Zu diesem Zweck wurde im Frühling 1979 von der Regionalverwaltung ein Seminar zu "Banjar, RT/RK ${ }^{17}$ in der Stadt Denpasar» durchgeführt. Deklariertes Ziel der Tagung war, Wege zu finden, um auch im urbanisierten Denpasar die traditionellen Werte und Normen der Bevölkerung zu bewahren. Augenfällig ist der mehrmalige Bezug im Seminardokument auf die Bedeutung intakter banjar (und damit einer intakten Kultur) für den Tourismus. ${ }^{18}$

Festgelegt wurden Integrationsbedingungen für neue Mitglieder in bereits bestehende banjar: Sowohl bei alten als auch bei neuen banjar sollte zwischen Voll- und Halbmitgliedern unterschieden werden. Angehörige beider Kategorien mußten erstens hinduistischen Glaubens und zweitens verheiratet sein. Vollmitglieder sollten zusätzlich Hausbesitzer sein, sich also dauerhaft niedergelassen haben, während die Halbmitglieder-Kategorie auf Mieter von Häusern oder Zimmern zugeschnitten blieb. Die Festlegung der Unterschiede in Rechten und Pflichten der beiden Kategorien wurde den jeweiligen banjar überlassen. Seit den frühen siebziger Jahren waren abernebst der Eingliederung einzelner Familien in bereits bestehende banjar - die zunehmenden Banjarneugründungen dominierendes Merkmal der Veränderung. Es mußten deshalb auch Struktur und Organisation neuzugründender banjar und deren Kompatibilität mit den tradierten banjar:Formen diskutiert werden. Im Seminar wurden letztere als banjar kuno (alt, orthodox) bezeichnet, um sie von der zweiten Art, den neuen banjar (banjar baru), abzugrenzen. Bei diesem neuen banjar-Typus handelt es sich v. a. um Zusammenschlüsse von Bewohnern in neu erschlossenen Gebieten, die auf diese Weise versuchen, in ihrer unmittelbaren Nachbarschaft gemeinsam solche Aspekte des Zusammenlebens zu regeln, die von staatlichen Institutionen (noch) nicht abgedeckt werden.

Im Seminar wurden folgende Kriterien für "korrekte» Neugründungen von banjar festgelegt: Als wichtigstes und unverzichtbares Element wurden Beziehungen zu einem desa adat und damit dessen Tempeln gefordert. Weiter muß er über ein Versammlungshaus, einen eigenen Tempel und einen kulkul (Wachturm mit hölzerner Schlitztrommel) verfügen, von einem Vorsteher geleitet 
sein und ein Reglement (awig-awig) besitzen. Von neuen banjar wurde deshalb gefordert, daß sie sich nach und nach obigen Kriterien anpassen und sich insbesondere um Aufnahme in ein desa adat bemühen sollten.

Der-modellhaftig vierstufige - Prozeß, der heute bei der Entstehung von banjar adat zu beobachten ist, illustriert den großen Einfluß der staatlichen Strukturen und Akteure auf den Bereich der Adatinstitutionen. In einer neuen Siedlung bildet sich zuerst eine lose konstituierte Gruppe (kelompok), die sich nach einiger Zeit in einen banjar baru umwandelt. ${ }^{19}$ Diesen wiederum führt die Verwaltung in einen banjar dinas über, welcher schließlich einem desa adat beitreten und sich damit zu einem «echten» banjar adat «aufwerten» kann.

Die hinter den gewählten Strategien und Maßnahmen sichtbar werdende Philosophie will zwar eine «moderne» indonesische Stadt, die aber ihren balinesischen Charakter nicht verlieren soll. Anzumerken ist schließlich, daß alle offiziellen Strategien wenig bis keinen Bezug auf die Resultate und komplexen Interpretationen der - mehrheitlich von Ausländern dominierten - Forschung nehmen. Die dort geführte Diskussion hat sich offensichtlich in weiten Stücken von den mehr praktisch-strategischen Bedürfnissen der balinesischen und indonesischen Verwalter abgekoppelt.

\section{Quantitative und qualitative Veränderungen von desa adat und banjar adat in Denpasar}

\section{Vorbemerkungen und Überblick}

Die Frage, wie viele der balinesischen Neuzuzüger - wie bei nichtbalinesischen Zuwanderern der Fall - nur den staatlichen Verwaltungs-(dinas-)Institutionen beitreten, und wie viele auch Mitglieder der banjar adat und desa adatwerden, ist aus verschiedenen Gründen schwierig zu beantworten. Dies ist zum ersten ein Definitionsproblem, denn es stellt sich die Frage, wie weit zurück jemand noch als Neueintretender bezeichnet werden soll, oder mit anderen Worten, ab wann eine Familie als asli («einheimisch») gelten kann. Zweitens stellt sich als Folge der Tatsache, daß einige - aber nicht alle - banjar zwischen verschiedenen Mitgliedsarten unterscheiden, ein Klassifizierungsproblem. ${ }^{20}$ Ebenso unterschiedlich sind die Eintrittskriterien in die verschiedenen banjar. Wichtigste Vorbedingungen sind Religionszugehörigkeit, $\mathrm{Zi}$ vilstand und Landbesitz. Nur wer balinesischer Hindu und verheiratet ist sowie Haus und Land besitzt, kann normalerweise dem banjar beitreten und damit automatisch Vollmitglied des desa adat werden. Es muß deshalb hier von einer Quantifizierung der Einzelbeitritte von Zuzügern in bestehende banjar und damit desa abgesehen werden. Einigermaßen verläßliche Zahlen hätte nur eine Befragung aller 344 banjar adat-Vorsteher ergeben, was von der Untersuchungsanlage her nicht möglich war. $^{21}$
Das in diesem Zusammenhang interessanteste Phänomen ist jedoch die weite Verbreitung von Doppelmitgliedschaften. Ausnahmslos wurde darauf hingewiesen, daß auch Zuzüger, die den lokalen Adatinstitutionen als Vollmitglieder (krama pangarep) beigetreten sind, weiterhin adat-Verbindungen zu ihrem Herkunftsbanjar und - desa pflegen, ja letzteren meist die größere Bedeutung beimessen. Dies gilt insbesondere für alle mit ihren Vorfahren und damit mit ihrem Ursprungspunkt (kawitan) zusammenhängenden Beziehungen und Riten. Solcherart haben dann Familien doppelte Verpflichtungen wahrzunehmen. Diese sich aus Doppelmitgliedschaften ergebenden Verpflichtungen (entweder persönliche aktive Teilnahme oder finanzielle Abgeltung) für beide desa halten denn auch viele Zuwanderer von einem Beitritt zur neuen desa adat-Gemeinschaft ab.

Im balinesischen Kontext stellt sich die zentrale Frage nach dem Ort, wo verstorbene Familienmitglieder beerdigt und kremiert werden. Obwohl dazu keinerlei Zahlen vorliegen, ist deutlich erkennbar, daß in solchen Fällen das Herkunfts desa klar bevorzugt wird. In Denpasar beispielsweise kommt es höchstens vor, daß Verstorbene so lange im dortigen Friedhof beigesetzt werden, bis die Familie über genügend Geld verfügt, um die Überführung des Leichnams ins Heimatdorf und die dort stattfindende Kremation zu bezahlen. Eine wichtige Rolle spielt sicherlich die geringe Fläche der Insel mit ihrer gut ausgebauten Verkehrsinfrastruktur. Diese gestattet nicht nur, den Leichentransport mit relativ geringem Aufwand realisieren zu können, sondern gewährt ebenso die Möglichkeit, relativ rasch ins Heimatdorf zu reisen und an den dortigen Zeremonien teilzunehmen.

Nach diesen Bemerkungen zu Einzelbeitritten liegt der Schwerpunkt in den folgenden Ausführungen bei den seit 1920 neugegründeten desa und banjar: Sie beziehen sich auf das gesamte Gebiet der heutigen kotamadya Denpasar, bestehend aus den drei Bezirken Denpasar West (Barat), Ost (Timur) und Süd (Selatan). Sie umschließt die eigentliche Kernstadt (kota oder hoofdstad der Kolonialzeit) und deren unmittelbares Hinterland, d. h. die 1978 in die Stadt eingemeindeten Dörfer.

Einen Überblick über die zahlenmäßigen Veränderungen von desa und banjar zwischen 1920 und 1986 innerhalb des gesamten heutigen Stadtgebietes vermittelt Tabelle 1:

Tab. 1 Desa und banjar adat 1920 und 1986.

\begin{tabular}{|l|r|l|c|l|r|}
\hline 1920 & \multicolumn{2}{|l|}{$\mathbf{1 9 8 6}$} & \multicolumn{2}{|l|}{ davon seit 1920 } \\
\hline desa adat & 29 & desa adat & 35 & alt & 28 \\
\hline & & & & neu & 7 \\
\hline banjar & 260 & banjar adat & 344 & alt & 253 \\
\hline & & & & neu & 91 \\
\hline
\end{tabular}

Quellen: 1920: KITLV: Collectie Korn, OR 435/157. 1986: Eigenerhebungen. 
In den 66 Jahren seit 1920 hat die Zahl der desa adat um 7 , diejenige der banjarum 91 zugenommen. Während bei den desa adat jede der 7 Neugründungen nachvollzogen werden kann, ${ }^{22}$ ist das Los einiger weniger banjar unklar. Dies betrifft diejenigen 7 der ursprünglich 260 banjar; die heute entweder nicht mehr unter demselben Namen existieren oder zu bestehen aufgehört haben. Während 3 dieser 7 banjar aufgelöst worden sind, war bei den restlichen 4 nicht mit Sicherheit festzustellen, ob und wann sie eventuell aufgelöst worden sind. Die folgenden Ausführungen sind deshalb unter diesem Vorbehalt zu betrachten.

\section{Neugründungen von desa adat}

Abb. 1 zeigt die 1986 existierenden 35 desa adat. Alle 7 neugegründeten desa adat entstanden durch Abspaltungen eines oder mehrerer banjar von bereits bestehenden Adatdörfern. In 4 Fällen geschah dies infolge von Konflikten, sei es zwischen jaba («gemeines Volk») und triwangsa (Adel) oder zwischen Alteingesessenen und Neuzuzügern. In den restlichen drei Fällen wurden Distanzen zu Tempeln und damit zur Verrichtung der religiösen Verpflichtungen als zu weit empfunden. Alle Neugründungen entstanden außerhalb der alten Kernstadt in

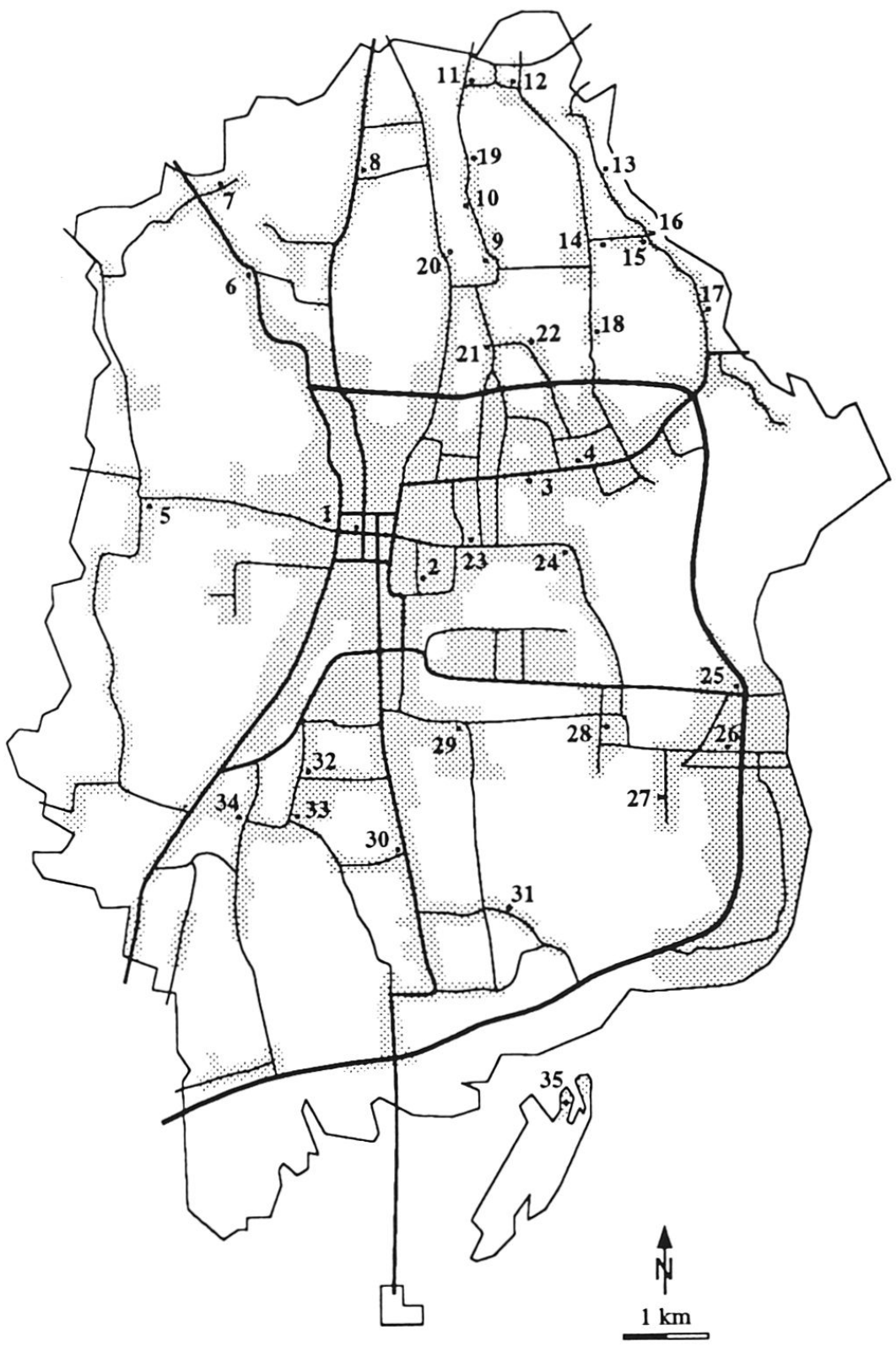

1 Badung/Denpasar

2 Yangbatu

3 Sumerta

4 Kesiman

5 Padangsambian

6 Ubung

7 Pohgading 1947/61

8 Peguyangan

9 Peninjoan

10 Kedua

11 Cengkilung

12 Anggabaya

13 Taman-Pohmanis 1981

14 Pinatih Puri 1937

15 Pinatih

16 Laplap 1961

17 Gunung-Buaji-Bekul 1963

18 Tembau

19 Jenah

20 Peraupan

21 Tonja

22 Oongan

23 Pagan

24 Tanjungbungkah 1953

25 Sanur

26 Intaran

27 Penyaringan 1920-1930

28 Renon

29 Panjer

30 Sesetan

31 Sidekarya

32 Pedungan

33 Kepaon

34 Pemogan

35 Serangan

Entwurf A.T. - Graphik R. Koechlin 1992 
Gebieten, die zumindest zum Zeitpunkt der jeweiligen Gründung noch nicht vom Urbanisierungsprozeß erfaßt worden waren.

Zuwanderung und der damit verbundene Bevölkerungsdruck scheinen - mit evtl. einer Ausnahme - bei allen Neugründungen keine oder nur eine untergeordnete Rolle gespielt zu haben. Insbesondere haben sich in keinem Fall Neusiedler zusammengeschlossen, um ein neues desa adat zu gründen. Wäre dies der Fall gewesen, hätten vorerst ein oder mehrere banjar gegründet werden müssen, die sich dann in einem zweiten Schritt als desa adat konstituiert hätten. Obwohl, wie unten gezeigt wird, Banjarneugründungen bzw. -abspaltungen häufig waren, haben sich im gesamten Untersuchungsgebiet keine dieser neuen banjar zu einem eigenständigen desa adat konstituiert. Der Urbanisierungsprozeß an sich hat also nicht direkt zu Desagründungen geführt.

Bei bereits bestehenden desa adat wurde organisatorischen Anpassungen - wie z. B. erleichterter Zugang von ganzen banjar - der Vorzug vor Abspaltungen oder Neugründungen gegeben. Die räumliche Ausdehnung des Dorfterritoriums (palemahan)eines desa adat scheint flexibel und die theoretisch mögliche Zahl der Mitglieder (pawongan) unbeschränkt zu sein, wie das Beispiel des größten "desa» adat, des hier als kota adat bezeichneten Denpasars, auf anschaulichste illustriert. Bevölkerungswachstum durch Zuwanderung kann so einerseits durch Ausweitung problemlos aufgefangen werden, andererseits ist damit aber ein gewisser Bedeutungs- und Authentizitätsverlust verbunden, wie die geringe Teilnahme an Zeremonien, insbesondere der kota adat Denpasar, zeigt.

Infolge der weitgehenden Reduktion des Funktionsbereichs von desa adat auf magisch-religiöse Belange muß den veränderten urbanen Rahmenbedingungen nicht im selben Ausmaß Rechnung getragen werden wie bei banjar; die wichtige säkulare Nachbarschaftshilfe-Funktionen erfüllen. Nicht zu unterschätzen ist sicherlich auch die Tatsache, daß die meisten balinesischen Zuwanderer den Kontakt zu ihrem Herkunftsdesa - auch bei allfälliger Desamitgliedschaft am neuen Wohnort - höher einschätzen, wie oben anhand der Wahl des Kremationsortes illustriert worden ist.

\section{"Anders als alle andern": kota adat Badung/Denpasar}

Das desa adat Badung, wie das heutige Denpasar früher korrekterweise noch genannt wurde, war schon als Sitz der zwei wichtigsten Zweige der Fürsten von Badung und erst recht seit 1906 als Zentrum der Kolonialverwaltung von Südbali das größte Adat«dorf» der ganzen Region und deshalb "lain dari yang lain» (anders als alle andern). Vor der niederländischen Inbesitznahme soll es insgesamt etwa 40 banjargezählt haben. Bis ins Jahr 1920 war die Zahl auf 57 angewachsen. ${ }^{23}$

Bis zum großen Erdbeben von 1917 befand sich der bale agung, in dem die Versammlungen (pasangkepan) abgehalten wurden, auf der Südseite der heutigen Jl. Gajah
Mada. Nach dem Erdbeben wurde die Straße verbreitert und so die pasarstraat, die "Chinatown" von Denpasar, geschaffen. Gleichzeitig wurde der bale agung auf die nördliche Seite der Straße in den mittleren Vorhof (jaba tengah) des Dorftempels verlegt. Bereits damals wurden keine Vollversammlungen aller desaadat-Mitglieder durchgeführt. Teilnehmer waren lediglich der bandesa als Vorsteher des desa adat, seine Assistenten, die klian banjarals Vertreter der banjar und der für den Unterhalt des Dorftempels verantwortliche pamangku sowie Spezialisten für die Zubereitung von Opfergaben. Heute ist Denpasar noch ausgeprägter "anders als alle andern" desa adat, weshalb es hier als kota adat (Adatstadt) bezeichnet wird - obwohl dieser Ausdruck von Denpasarern selbst nicht verwendet wird. Seit 1920 sind 23 neue banjar inkorporiert worden, womit die kota adat auf 80 banjar angewachsen und sicherlich die größte Adatgemeinde auf Bali ist. Sie scheint auch das einzige "desa»adat zu sein, dem auch Mieter beitreten können, m. a. W. Land- und Hausbesitz keine notwendige Voraussetzung zur Mitgliedschaft sind.

Im Verständnis der Dorfvorsteher setzt sich der krama desa von Denpasar nicht aus Einzelmitgliedern zusammen, sondern aus den banjar; in Denpasar tempekan desa genannt. Beim Verkehr mit ihnen bedient sich das desa adat ausschließlich administrativer Dinaskanäle. Briefe und Mitteilungen werden über die lurah und kepala desa an die klian dinas weitergeleitet, welche dann den klian adat oder auch die Adatabteilung (seksi keadatan) innerhalb der banjar informieren. Ähnlich ist das Bild bei der jeweils nach dem odalan stattfindenden Versammlung (pasangkepan)im pura desa. Nebst den klian adatsind die lurah, die kepala desa sowie die klian dinas als voll beschlußfähige Teilnehmer eingeladen. Analoge Organisationsformen sind auch in den beiden anderen großen desa adat Kesiman und Sumerta zu finden. Die Präsenz an den Versammlungen ist bescheiden. Selten treffen sich mehr als 40 Teilnehmer, weshalb denn auch der Versammlungs "pavillon» (bale pasangkepan)mehr als genug Platz bietet.

Die kota adat Denpasar dürfte die einzige Adatgemeinschaft auf Bali sein, welche die jährlich wiederkehrende "Geburtstagsfeier" (odalan) des Dorftempels auf drei aufeinanderfolgende Tage verlängert hat. Diese Neuerung wurde in den 60er Jahren in der Absicht eingeführt, allen banjar die Gelegenheit zur Teilnahme zu bieten. Der anfängliche Plan, jeweils pro Tag einem perbekelan und seinen banjar den Zugang zu gewähren, mußte bald aufgegeben werden, da sich die banjar nicht an diese Bestimmung hielten. So ist es denn heute jedem banjar und seinen Mitgliedern freigestellt, an welchem der drei Tage sie den Tempel besuchen wollen. Insgesamt nehmen aber weniger als die Hälfte der 80 banjar aktiv am odolan im eigentlichen Sinne des «Die-Last-Tragens» (nyungsung) teil. Unter den wirklich aktiven befinden sich vor allem die alten, im Stadtzentrum gelegenen banjar; während von den später ins desa eingetretenen häufig nur gerade der klianteilnimmt. Eine Ausnahme bilden aus dem Zen- 
trum in periphere banjar ausgesiedelte "Stadt-Badunger", die sich ihrem Ursprungs-desa adat weiterhin verpflichtet fühlen. Kostenbeiträge ans odalan sind denn auch freiwillig und hängen von der Spendefreudigkeit der Gläubigen ab.

Viele der in Denpasar niedergelassenen Zuwanderer fühlen sich - obwohl sie u. U. Vollmitglieder eines der banjar adat sind - dem desa adat nicht sonderlich verbunden. Es scheint, daß ihre Loyalität gegenüber dem banjar wichtiger ist, da seine (profane) Nachbarschaftsfunktion für das Alltagsleben eine größere Rolle spielt. Für (sakrale) Desafunktionen fühlen sie sich weiterhin dem Herkunftsort verpflichtet. Bei Diskussionen wird häufig darauf verwiesen, daß Zuwanderer ungern den "fremden" Dorftempel benützen, da sie sich unwohl (malu) fühlen würden. Wie oben erwähnt, werden Verstorbene nicht in Denpasar kremiert, sondern höchstens so lange im Friedhof bestattet, bis sie ins Herkunfts desa zur Kremation übergeführt werden können. Bei Beerdigungen und Exhumierungen gewähren denn auch weniger Mitglieder des Wohnbanjar als Delegationen aus dem Herkunftsbanjar nachbarschaftliche Hilfe.

\section{Neugründungen von banjar}

Als erstes muß festgestellt werden, daß heute die Territorien der einzelnen banjar nur mehr teilweise "intakt» sind, wie sie es entsprechend ihrer idealtypischen Funktion als Nachbarschaftsvereinigung sein müßten. Bei beinahe $64 \%$ der banjar adat und banjar baru ist die Bevölkerung "durcheinander vermischt" (seluk-menyeluk), sind banjar also wohl soziopolitische Gruppierungen, nicht aber Wohneinheiten mit klaren Grenzen. Interessanterweise ist dies auch bei knapp der Hälfte der banjar dinas der Fall, die sich in dieser Hinsicht anscheinend ihren Adatvarianten anpassen mußten. Immer häufiger werden deshalb heute Straßennamen an Stelle von banjar zur räumlichen Orientierung benützt, wobei dies eher bei Neuzuzügern als bei alteingesessenen «Stadt-Denpasarern" der Fall sein dürfte. ${ }^{24}$

Aufgeschlüsselt nach Abspaltungen von bereits bestehenden banjar und vollständigen Neukonstituierungen verteilen sich die 91 neuen banjar folgendermaßen auf die vier gewählten Betrachtungsperioden:

Tab.2 Abspaltungen und Neukonstituierungen von banjar.

$\begin{array}{lrc}\text { Periode } & \frac{\text { Abspal- }}{\text { tungen }} & \frac{\text { Neukonsti- }}{\text { tuierungen }} \\ 1925-1940 & 11 & 2 \\ 1941-1955 & 12 & 4 \\ 1956-1970 & 4 & 9 \\ 1971-1985 & 7 & 42 \\ \text { Total } & 34 & 57\end{array}$

Wie Abb. 2 zeigt, korrelieren die Zahlen der Tab. 2 gut mit der jeweiligen Anzahl der zwei Typen von banjar; den «klassischen" banjar adat und den (neuen) banjar baru. Neugründungen von "echten" banjar adat dominierten die beiden ersten Betrachtungsperioden bis 1955. Später spielte Zuwanderung die Hauptrolle bei Gründungen, womit der "klassische» Typus vom neuen Typus des «suburbanen" banjar abgelöst wurde (auch wenn letztere nicht nur in vorstädtischen Gebieten entstanden). Der neue "desa adat-lose» Banjartypus stellt denn auch die mit Abstand größte Gruppe innerhalb einer Betrachtungsperiode und zeigt klar den Trend zukünftiger Entwicklungen. In der letzten Periode sind von den insgesamt 42 neukonstituierten banjar nur 4 bereits zu banjar adat aufgewertet worden.

Abb. 2 Banjar adat versus banjar baru (neue banjar).

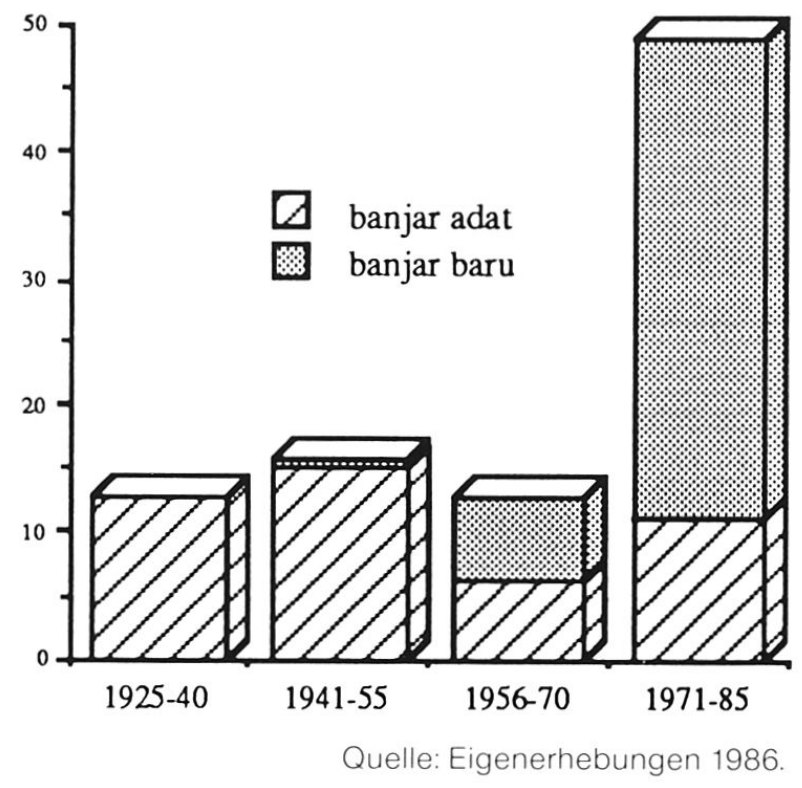

Bei alten, etablierten banjar machen sich im Gegenzug Abschottungstendenzen bemerkbar. Einerseits werden in gewissen banjar keine neuen Mitglieder aufgenommen, und andererseits sind vermehrt Exklusivbanjar gegründet worden. Beide Phänomene dürfen wohl als Reaktion auf den zunehmenden Bedeutungsverlust autochthoner Badung-Familien gegenüber Zuwanderern gewertet werden.

Nebst den erwähnten quantitativen Veränderungen machten die ursprünglich aus einer ländlichen Gesellschaft hervorgegangenen Institutionen desa und banjar auch einen Funktions- und Strukturwandel durch. Dies erstaunt nicht: Da auf dem Land die Gemeinschaft überschaubar und die interpersonelle Kommunikation intensiv ist, genießen hier auch traditionelle Werte der Solidarität (und damit Institutionen, die diese stützen) einen höheren Stellenwert. Eine städtische Gesellschaft mit hohem Anteil an Zuwanderern ist demgegenüber anony- 
mer, und für Kommunikation und Interaktion spielen wohnortspezifische Gemeinschaften, wie es sowohl desa und banjar sind, eine weit geringere Rolle. Städtische desa und banjar können somit nur mehr beschränkt mit ihren dörflichen Pendants verglichen werden. Der Bedeutungsverlust der endogenen Adatinstitutionen findet seinen Niederschlag im von Banjar- und Desaverantwortlichen beklagten Desinteresse an gemeinschaftlichen Aktivitäten und einem Mangel an aktiven Mitgliedern.

In diesem Zusammenhang steht auch die Tatsache, daß zwar einerseits die Anzahl von Abspaltungen infolge von Konflikten abgenommen hat, andererseits aber angenommen werden kann, daß sich die Konflikthäufigkeit in der Stadt insgesamt kaum verringert hat. Ein Ausdruck des Verschiebens von Konfliktfeldern im urbanen Kontext kann darin gesehen werden, daß «domestizierte» und «ästhetisierte» banjar mit nivellierten Reglementen (awig-awig) ihre Bedeutung als Ort der Austragung fundamentaler Konflikte verloren haben. Letztere lassen sich heute eher aus den Leserbriefspalten der Regionalzeitung Bali Post isolieren und betreffen weniger Fragen des adat als vielmehr seiner modernen Pendants, der mehr schlecht als recht funktionierenden Institutionen und Organe der staatlichen Verwaltung. Standen früher Kremationen im Zentrum der Banjaraktivitäten, so sind heute im urbanen Alltag des modernen bürokratischen Staats zunehmend administrative (dinas-)Funktionen gefragt. «Moderne» Formen der Solidarität - zwischen Arbeitskollegen oder beispielsweise über staatliche Beamtenversicherungen - gewinnen auch in Denpasar auf Kosten traditioneller Nachbarschaftssolidarität an Bedeutung.

\section{Schlußfolgerungen}

Im Bezug auf städtische "desa»adat kann zwar einerseits festgestellt werden, daß sie gegenüber dem Urbanisierungsproze $\beta$ insofern weitgehend «immun» $\mathrm{zu}$ sein scheinen, da sie sich in Größe des Territoriums und Mitgliedszahl als anpassungsfähig erweisen - im Gegensatz zu banjar, die davon sehr direkt betroffen sind. Andererseits kann heute von einer umfassenden Bedeutung der Adatinstitution desa für die Stadtgesellschaft nicht die Rede sein, hat mithin eine kota adat wenig mit einem dörflichen desa adat gemeinsam. Ohne die administrativen Kanäle und behördlichen Repräsentanten, wie klian dinas und lurah oder kepala desa, wären die kota adat Denpasar und andere große desa adat wie Kesiman und Sumerta vermutlich schon längst "unregierbar». Wie die Besucherfrequenz an den jeweiligen Zeremonien zeigt, scheint der von den provinziellen Religionsverwaltern kontrollierte Tempel pura agung Jagat Natha die in der urbanen, «anonymen» Lebenssituation nachgefragten religiösen Bedürfnisse besser zu befriedigen als der pura de sa der lokalen Adatgemeinschaft von Denpasar.

Urbane banjarsind bedeutend heterogener als ihre idealtypischen ländlichen Vorbilder. Zum Typus des neuen banjar baru zählen 1986 zwar insgesamt erst gut 13\% aller banjar auf dem Gebiet der kota administratif. Da sie unter den in jüngerer Zeit gegründeten jedoch bei weitem in der Mehrheit sind, dürfte sich dieser Trend in Zukunft noch weiter verstärken. Diese moderne Variante der banjar ist deshalb bedeutsam, weil sie das institutionelle Äquivalent des (Sub-)Urbanisierungsprozesses darstellt, der die Mitglieder der banjar in ihr neues Wohnquartier gebracht hat. Die neuen Stadtbanjar lassen sich mit Quartiervereinen in europäischen Städten vergleichen, die für ihre Mitglieder vor allem "weltliche» Funktionen erfüllen, welche von keiner staatlichen Institution abgedeckt werden.

Daß der Begriff banjar baru von offizieller Seite eingeführt wurde, illustriert, daß auch von Beamten die nicht mehr dem Idealtypus entsprechende Funktion und Struktur dieser neuen Institutionen wahrgenommen wird. Das Konzept der banjar baru ist in diesem Zusammenhang als Versuch zu werten, das Fortbestehen dieser Institution auch im urbanen Kontext zu gewährleisten. Das behördliche Drängen auf einen Beitritt der banjar baru ins desa adat soll das gesellschaftliche Idealbild eines intakten adat und damit einer intakten Kultur aufrechterhalten. Es zeigt sich aber, daß sich banjar baru wenn überhaupt - erst nach gewisser Zeit ins desa adat integrieren und auch ohne letzteres funktionsfähig sind. Folgt man den idealtypischen Konzepten sowohl westlicher als auch balinesischer Provenienz, so ergibt sich demzufolge die paradoxe Situation, daß banjar zwar theoretisch desa untergeordnet sein sollten, erstere aber trotzdem ohne desa existieren können, während desa ohne banjar nicht denkbar sind.

Interessant am beschriebenen Prozess der Banjarbildung ist, daß seit der Kolonialzeit eine Umkehrung der Reihenfolge der einzelnen Stufen stattgefunden hat. Während die kolonialen Behörden den bestehenden banjar eine neue Dinasvariante zur Seite stellten, werden heute banjar dinas zu banjar adat "aufgewertet». Damit entstehen eigentlich wiederum banjar von vorkolonialem Zuschnitt, in denen die Trennung von Adat-und Dinasbereichen und -funktionen wenn auch nicht völlig aufgehoben, so doch minimiert ist, und eine Institution die anfallenden Probleme bewältigen kann. Eine gewisse Ironie der Geschichte liegt in der Tatsache, daß somit der tiefgreifendste koloniale Eingriff - die Dichotomisierung in Adat- und Dinasinstitutionen - rückgängig gemacht wird. Diese neuen banjar sind damit - wie in vorkolonialer Zeit - wiederum Gemeinschaftsorganisationen, die nebst den magisch-spirituellen Beziehungen zu den Vorfahren auch die profanen Beziehungen zwischen Volk und den neuen Herrschern, der staatlichen Verwaltung, regeln.

Im städtischen Kontext bedeutsam ist schließlich die Tatsache, daß bei der offiziellen Diskussion der Adatinstitutionen von vornherein alle nichtbalinesischen Immigranten (und nichthinduistischen Balinesen) von den banjar und damit desa adat ausgeschlossen sind - was früher nicht unbedingt der Fall war, da insbesondere Chi- 
nesen oft Mitglieder lokaler banjar adat waren. Die Summe aller Desa- und damit Banjarmitglieder macht ja keinesfalls die "Stadtgesellschaft" aus. Städtische desa und banjar haben somit ihren Alleinvertretungsanspruch aller Bewohner ihres Territoriums verloren. Sie sind - ob alt oder neu - nur für Angelegenheiten ihrer Mitglieder zuständig, womit die Gefahr der Entwicklung zu einer "Zweiklassen"-Gesellschaft von banjar- und desa adatfähigen Balinesen einerseits und übrigen Stadtbewohnern andererseits besteht. Ob diese Entwicklung allerdings von großer Tragweite ist, bleibt abzuwarten. Dies hängt insbesondere davon ab, ob der hier - zumindest für den städtischen Kontext - postulierte Bedeutungsverlust des adat und seiner Institutionen als gemeinsame soziale, rechtliche, moralische und religiöse Basis der balinesischen Gesellschaft weiter fortschreitet.

Trotzdem sehen sich Verwaltung und mit ihr affiliierte Akteure in (halb)staatlichen Religions- und Adatorganisationen der Herausforderung gegenüber, Wege und realisierbare Strategien zu finden, um auch andere Gruppen in die "Stadtgemeinschaft" zu integrieren - liegt doch in Ausgrenzungen der oben beschriebenen Art ein erhebliches Konfliktpotential, gerade in einer Zeit zunehmender religiöser Spannungen, bei denen die Position der kleinen hinduistischen Minderheit im vom Islam dominierten Gesamtindonesien nicht gerade die stärkste ist.

\section{Anmerkungen}

' Vgl. dazu SCHULTE NORDHOLT (1985) und LEEMANN, TARNUTZER, WÄLTY (1987), wo die Genese dieses idealisierenden Balibildes als Antithese des durch den Islam seinen hinduistischen Wurzeln "entfremdeten" Java diskutiert wird.

${ }^{2}$ Vgl. TARNUTZER (1933: 1 ff.). Studien (ausländischer Forscher) über urbane Entwicklung lassen sich immer noch an einer Hand abzählen. Zu den wenigen Ausnahmen zählen C. GEERTZ (1963), COUTEAU (1988), MCTAGGART (1980), TARNUTZER (1993). Die umfassende Bibliographie von STUART-FOX (1992: $131 \mathrm{ff}$.) listet denn auch unter urban areas fast ausschließlich Pläne und Berichte verschiedener Behörden auf.

${ }^{3}$ DEPARTEMENT VAN ECONOMISCHE ZAKEN (1936); KANTOR STATISTIK PROPINSI BALI (1990: 36).

${ }^{4}$ RUTZ (1987: 130).

${ }^{5}$ Die hier ausschnittweise vorgestellten Resultate sind während Forschungsaufenthalten zwischen 1983 und 1986 erhoben worden. Unterstützung gewährten: Schweizerischer Nationalfonds (Nachwuchsstipendium), Stiftung für wissenschaftliche Forschung an der Universität Zürich, Jubiläumsspende der Universität Zürich und STEO-Stiftung, Zürich.

${ }^{6}$ Vgl. Artikel von A. Leemann in dieser Nummer (GH Nr. 1/93) S. 6-18.

Beide endogenen Institutionen existieren seit vorkolonialeŕ Zeit. Quellen zu dieser Phase sind jedoch spärlich, weshalb ihre genauen historischen Wurzeln unklar bleiben. Vgl. insbesondere WÄLTY (in Vorb.)

${ }^{8}$ Vgl. GUERMONPREZ (1980: 48 ff.), SCHAAREMAN (1986: 82)

${ }^{9}$ Vgl. insbesondere WARREN (1990) und PARKER (1989).

\section{Vgl. TARNUTZER (1993: 155 ff.)}

Zur Diskussion dieser im vorkolonialen Südostasien dominierenden Staatsform siehe NAS (1986: 18). Zur Geschichte des vorkolonialen Badung siehe TARNUTZER (1993: $11 \mathrm{ff}$.); SCHULTE NORHOLT (1980).

${ }^{12}$ VAN KOL (1914: 402; Fußnote 1). Die Kolonialarmee verzeichnete nur vier Tote.

${ }^{13}$ Bal.: puputan, im übertragenen Sinn: "bis zum letzten Mann".

${ }^{14}$ Abgeleitet vom niederländischen Wort "dienst». Anfänglich wurden dafür auch andere Bezeichnungen verwendet.

${ }^{15}$ Mit dem Begriff indonesianisasi werden jene Strategien bezeichnet, mit denen die große regionale und lokale Variationsbreite des adat nivelliert und gesamtindonesisch vereinheitlicht werden soll. Eine direkte Folge und teilweise auch Reaktion auf die indonesianisasi sind regionale Vereinheitlichungsbestrebungen des adatund seiner Institutionen, für die analog der Begriff balisasi benutzt wird (TARNUTZER 1993: $183 \mathrm{ff}$.).

${ }^{16}$ Entscheidende Schritte auf dem Weg zur Vereinheitlichung der desa adatwurden auf zwei Konferenzen in Denpasar 1969 und 1974 gemacht (TARNUTZER 1993: 183 ff.).

RT: rukun tetangga, RK: rukun kampung: Nachbarschaftsund Quartierorganisationen javanischen Zuschnitts mit administrativer (dinas-)Funktion.

${ }^{18}$ FAKULTAS HUKUM DAN PENGETAHUAN MASYARAKAT (1979: $46 \mathrm{f}$.)

${ }^{19}$ Zur Zeit der Untersuchung (1986) existierten in Denpasar neun dieser kelompok.

${ }^{20}$ Neben Vollmitgliedern (pangarep) existiert in manchen, vor allem älteren banjar die Kategorie der "Pensionierten" (pangele) für Mitglieder, deren Söhne sich verheiratet und dann ihre Nachfolge angetreten haben. In einigen banjar wird diese Kategorie auch auf Neuzuzüger angewendet. Letztere werden aber auch wahlweise als anggota tamu (Gast), penyumbang ("Supporter»), penumpang (Passagier. Gast) oder penyokong (Sponsor) bezeichnet - interessanterweise alles indonesische und nicht balinesische Ausdrücke. Schließlich gibt es banjar, welche nur eine Mitgliedskategorie kennen.

Die Vorsteher (bandesa) der großen desa adat (Denpasar, Sumerta, Kesiman) konnten keine Angaben machen, da sie nicht in Kategorien von Einzelmitgliedern, sondern ganzer banjar zu denken gewohnt und ihre desa adat zu groß sind, als daß sie noch den Überblick über die Lage in den einzelnen banjar hätten.

22 Für Details siehe TARNUTZER (1993: $200 \mathrm{ff}$.).

${ }^{23}$ KITLV: Collectie Korn, OR 435/157.

${ }^{24}$ Schon zu kolonialer Zeit war dies bei den damals beiden einzigen mit Namen versehenen Straßen (der School- und Pasarstraat) der Fall, während für das übrige Stadtgebiet noch ausschließlich banjar als Adressen dienten (pers. Mitteilung des ehem. Kolonialbeamten C. J. Grader).

\section{Literatur}

COUTEAU, J. (1988): Regards sur Denpasar. In: Archipel (36): 43-58.

DEPARTEMENT VAN ECONOMISCHE ZAKEN (1936): Volkstelling 1930; Deel V.: Inheemsche Bevolking van Borneo, Celebes, de kleine Soenda eilanden en de Molukken. Batavia: Landsdrukkerij. 
FAKULTAS HUKUM DAN PENGETAHUAN MASYARAKAT (1979): Pengorganisasian kelompok-kelompok masyarakat ditinjau dari segi hukum adat: Seminar tentang banjar, RT/ RK di kota Denpasar, 10 Maret 1979. Denpasar: Universitas Udayana.

GEERTZ, C. (1963): Peddlers and Princes: Social Development and Economic Change in Two Indonesian Towns. Chicago: University Press

GUERMONPREZ, J.F. (1980): L'organisation villageoise à Bali. Essai de mise en perspective. In: Asie du Sud-Est et Monde Insulindien (11) 1-4:37-54

KANTOR STATISTIK PROPINSI BALI (1990): Statistik Bali: 1989. Denpasar.

KOL, H. H.van (1914): Driemaal dwars door Sumatra en zwerftochten door Bali. Rotterdam: Brusse.

LEEMANN, A.; TARNUTZER, A.; WÄLTY, S. (1987): Bali: Touris mus und Entwicklung. In: Wirtschaft, Kultur und Entwicklung 8. Tübinger Gespräch zu Entwicklungsfragen: Materialien zum Internationalen Kulturaustausch (28): 179-196. Stuttgart: Institut für Auslandsbeziehungen.

McTAGGART, D. W. (1980): An Evaluation of the Master Plan for Singaraja, Bali. In: Malaysian Journal of Tropical Geography (1): 21-33.

NAS, P. J. M. (1986): The Early Indonesian Town. Rise and Decline of the City-State and its Capital. In: Peter J. M. Nas (ed.) The Indonesian City. Dordrecht: Foris.

PARKER, L. (1989): Village and State in "New Order" Bali. Canberra: PhD Thesis Australian National University.
RUTZ, W. (1987): Cities and Towns in Indonesia; their development, current positions and functions with regard to administration and regional economy. Stuttgart: Bornträger.

SCHAAREMAN, D. (1986): Tatulingga: Tradition and Continuity: An Investigation in Ritual and Social Organization in Bali (Basler Beiträge zur Ethnologie, Bd. 24). Basel: Ethnologisches Seminar und Museum für Völkerkunde.

SCHULTE NORDHOLT, H. (1980): Macht, Mensen en Middelen: Patronen van Dynamick in de Balische Politiek 1700-1840. Amsterdam, Doktoraalscriptie, Freie Universität.

SCHULTE NORDHOLT, H. (1985): Paradox, contradictie, crisis: Koloniale beeldvorming en politiek conflict in Bali. (Bijdrage Kota VI: laat koloniale en postkoloniale staat in Azië, Rotterdam August 1985.)

STUART-FOX, D. (1992): Bibliography of Bali. Publications from 1920 to 1990. Leiden: KITLV Press.

TARNUTZER, A. (1993): Kota Adat Denpasar (Bali): Stadtentwicklung, staatliches Handeln und endogene Institutionen Zürich: Dissertation Universität Zürich.

WÄLTY, S. (in Vorb.): Kintamani: Dorf, Landbesitz und Feiertage. Institutionen im Entwicklungsprozeß in einer peripheren Region auf Bali. Zürich: Dissertation Universität Zürich.

WARREN, C. (1990): Adat and Dinas: Village and State in Contemporary Bali. Perth: PhD Thesis University of Western Australia. 